\title{
A Potato Peel Extract Stimulates Type I Collagen Synthesis via Akt and ERK Signaling in Normal Human Dermal Fibroblasts
}

\author{
Mari Suto, ${ }^{*}, a$ Hirofumi Masutomi, ${ }^{a}$ Katsuyuki Ishihara, ${ }^{a}$ and Hitoshi Masaki ${ }^{b}$ \\ ${ }^{a}$ Calbee, Inc.; R\&D Center, 23-6 Kiyohara-Kogyodanchi, Utsunomiya, Tochigi 321-3231, Japan: and \\ ${ }^{b}$ Tokyo University of Technology; 1404-1 Katakura-machi, Hachioji, Tokyo 192-0982, Japan. \\ Received February 26, 2019; accepted June 11, 2019
}

The ability of dermal fibroblasts to synthesize collagen decreases with ages. The integrity of collagen fibers severely decreases in aged skin, causing its characteristic morphological changes such as wrinkles and sagging. To prevent and improve skin aging, the stimulation of collagen synthesis in dermal fibroblasts is important. Potato peels contain many biofunctional compounds, but not much is known about their effects on human skin physiology. To characterize the potential effects of a potato peel extract (PPE) against skin aging, we examined its effects on the synthesis of type I collagen by normal human dermal fibroblasts (NHDFs). Treatment with the PPE significantly increased the expression of type I collagen mRNA in NHDFs and their secretion of type I collagen. To elucidate the mechanism involved, we examined the signaling pathway controlled by transforming growth factor- $\beta$ (TGF- $\beta$ ), which regulates the synthesis of type I collagen. Treatment of NHDFs with the PPE significantly increased the expression of TGF- $\beta$ receptor mRNA. TGF- $\beta$ signaling involves Smad-dependent and Smad-independent pathways, like phosphatidylinositol-3 kinase (PI3K)/Akt and mitogen-activated protein kinase (MAPK)/extracellular signal-regulated kinase (ERK). The PPE did not activate Smad, but significantly activated Akt and ERK. These results demonstrate that the PPE activates PI3K/Akt and MAPK/ERK signals via TGF- $\beta$ receptors, which stimulate the synthesis of type I collagen in NHDFs. These results suggest that the PPE could be a novel and effective antiaging material.

Key words potato; collagen; dermal fibroblast; transforming growth factor- $\beta$; Akt; extracellular signal-regulated kinase (ERK)

\section{INTRODUCTION}

The dermis, which is located beneath the epidermis, is deeply involved in regulating the firmness and elasticity of the skin. The dermis is constructed of structural elements, including collagen fibers, elastin fibers and proteoglycans, that are produced by dermal fibroblasts. Among those, collagen fibers, which provide the structural skeleton of the dermis, play a key role in maintaining the mechanical strength and flexibility of the skin. ${ }^{1,2)}$ In aged skin, a drastic depletion of collagen fibers at papillary regions has been recognized as a typical characteristic that results in wrinkling and sagging. ${ }^{3,4)}$ The appearance of wrinkles and sagging strongly influences the QOL, because of their impact on visible appearance. Therefore, suppressing and/or improving wrinkling and sagging by stimulating collagen synthesis is an important approach to maintaining a high QOL. Collagen fibers in the dermis are primarily composed of types I and III collagen. Because type I collagen represents more than $90 \%$ of total collagen, ${ }^{5)}$ it is an important target to prevent and improve skin wrinkling and sagging.

The synthesis of type I collagen has been reported to be controlled by two main growth factors, insulin-like growth factor-1 (IGF-1) $)^{6)}$ and transforming growth factor- $\beta$ $(\text { TGF- } \beta)^{7,{ }^{78)}}$ IGF-1 binds to the IGF-1 receptor, a transmembrane tyrosine kinase, and transmits signals by modulating the activity of its adaptor molecules (Insulin receptor substrate-1, Src homology 2 domain containing). The downstream signaling of this cascade is known to involve the phosphatidylinositol-3 kinase (PI3K)/Akt pathway and the mitogen-activated protein kinase (MAPK)/extracellular signal-related kinase
(ERK) pathway. ${ }^{910)}$ On the other hand, TGF- $\beta$ binds to a heterocomplex formed by two types of receptors, TGF- $\beta$ type I receptor (T $\beta$ RI) and TGF- $\beta$ type II receptor (T $\beta$ RII) in order to transmit intracellular signals. TGF- $\beta$ signal transmission is known to involve Smad-dependent and Smad-independent pathways. ${ }^{11)}$ In the Smad-dependent pathway, TGF- $\beta$ causes the phosphorylation of receptor-activated Smad (R-Smad). Phosphorylated R-Smad forms a complex with commonmediator Smad (Co-Smad) after which it translocates into the nucleus, where it functions as a transcription factor ${ }^{7,8)}$ that can upregulate the transcription of genes involved in type I collagen synthesis. On the other hand, the Smad-independent pathway involves stimulation by TGF- $\beta$ causing the induction of signaling molecules like PI3K/Akt, MAPK and Rholike guanosine $5^{\prime}$-triphosphatase (GTPase).$^{11-13)}$ These factors control the synthesis of type I collagen through a pathway distinct from the Smad-dependent pathway. However, IGF-1 and TGF- $\beta$ are important growth factors in both pathways that control the synthesis of type I collagen in fibroblasts.

The potato (Solanum tuberosum L.) is among the five most significant food crops of the world. The edible portion of the plant, the tuber, contains many nutritional ingredients, including ascorbic acid, ${ }^{14)}$ folic acid $^{15)}$ and potassium. ${ }^{16)}$ Although the peel of each potato is removed during processing, the interest of reducing industrial byproducts like the potato peel to aim for zero emissions is currently becoming a global fashion. Thus, we considered how to effectively use a potato peel since some functional ingredients, including phenolic compounds such as chlorogenic acid, and flavonoids such as catechin, have been identified in potato peels. ${ }^{17-19)}$ However, 
there have been relatively few reports on the functionality of those ingredients on the skin. For instance, previous research studies have reported that ascorbic acid ${ }^{20)}$ and some phenolic compounds and flavonoids ${ }^{21)}$ influence the synthesis of type I collagen in the dermis. Ascorbic acid is well known to stimulate collagen synthesis by contributing to the formation of its triple helix structure by promoting the hydroxylation of proline and lysine in procollagen. ${ }^{20)}$ On the other hand, phenolic compounds and flavonoids have been reported to suppress the synthesis of matrix metalloproteinases (MMPs) through their antioxidant activities. ${ }^{22-24)}$ Those reports suggest that a PPE also might be capable of preventing and/or improving skin aging through effects on collagen.

Thus, the purpose of this study was to investigate the potential of a potato peel on the synthesis of type I collagen using normal human dermal fibroblasts (NHDFs) expecting to achieve zero emission and to propose an effective use of this byproduct.

\section{MATERIALS AND METHODS}

Materials NHDFs were obtained from Kurabo (Osaka, Japan). Dulbecco's modified Eagle's medium (DMEM) was purchased from Sigma-Aldrich (St. Louis, MO, U.S.A.). Fetal bovine serum (FBS) was purchased from Biowest (Nuaillé, France). Trypsin-ethylenediaminetetraacetic acid (EDTA) was purchased from Gibco $^{\mathrm{TM}}$ Invitrogen Corporation (Carlsbad, CA, U.S.A.). Human type I collagen was obtained from Kyowa Pharma Chemical (Toyama, Japan). Anti-collagen I was obtained from Cosmo Bio (Tokyo, Japan). Streptavidinhorseradish peroxidase (HRP) was purchased from R\&D Systems (Minneapolis, MN, U.S.A.). ABTS was purchased from FUJIFILM Wako Pure Chemical Corporation (Osaka, Japan). cOmplete, Mini, EDTA-free, Protease Inhibitor Cocktail and PhosSTOP was purchased from Sigma-Aldrich. IGF-1 Receptor $\beta$ (D23H3) XP Rabbit monoclonal antibody (mAb), Phospho-IGF-1 Receptor $\beta$ (Tyr1135) (DA7A8) Rabbit mAb, Smad3 (C67H9) Rabbit mAb, Phospho-Smad3 (Ser423/425) (C25A9) Rabbit mAb, Akt (pan) (C67E7) Rabbit mAb, Phospho-Akt (Ser473) (D9E) XP Rabbit mAb, p44/42 MAPK (Erk1/2) (137F5) Rabbit mAb, Phospho-p44/42 MAPK (Erk1/2) (Thr202/Tyr204) (D13.14.4E) XP Rabbit mAb, $\beta$-Actin Antibody, Anti-rabbit immunoglobulin $\mathrm{G}$ ( $\mathrm{IgG}$ ) and HRP-linked Antibody, U0126 were purchased from Cell Signaling Technology (Danvers, MA, U.S.A.). LY294002 was purchased from FUJIFILM Wako Pure Chemical Corporation.

Preparation of the Potato Peel Extract (PPE) Potato (Solanum tuberosum L. c.v. Kitahime) peels were collected and dried at $60^{\circ} \mathrm{C}$, after which they were soaked in $50 \%$ ethanol for 1 week. After filtration, the solvent was evaporated using a centrifugal evaporator (NVC-1100, EYELA, Tokyo, Japan) to prepare the PPE, which was stored at $-80^{\circ} \mathrm{C}$ until used.

Cell Culture NHDFs were cultured in DMEM containing $5 \% \mathrm{FBS}$ at $37^{\circ} \mathrm{C}$ and $5 \% \mathrm{CO}_{2}$. After harvesting with $0.25 \%$ Trypsin-EDTA, cells were inoculated in 96-well plates or in $60-\mathrm{mm}$ dishes at concentrations of $2.0 \times 10^{4}$ cells/well and $80 \times$ $10^{4}$ cells/dish, respectively, and then used for these experiments.

Enzyme-Linked Immunosorbent Assay (ELISA) NHDFs were cultured in DMEM containing 0.5\% FBS with or without the PPE at various concentrations for $24 \mathrm{~h}$. Afterwards, the culture supernatants were collected and amounts of type I collagen, TGF- $\beta 1$ and IGF-1 were quantified using
ELISAs. The ELISA for type I collagen was conducted as follows: The culture supernatants were coated in 96-well plates and were incubated at $4{ }^{\circ} \mathrm{C}$ for $24 \mathrm{~h}$. After blocking with $1 \%$ bovine serum albumin (BSA) in phosphate buffered saline $(\mathrm{PBS})(-)$ at room temperature, the plates were incubated with the primary antibody at $4^{\circ} \mathrm{C}$ for $24 \mathrm{~h}$ and then were further incubated with the secondary antibody for $1 \mathrm{~h}$. Antibodies bound to type I collagen on the plate were visualized with an ABTS coloring solution, and absorbances were measured at $405 \mathrm{~nm}$ using a microplate reader (Spark 10M, Tecan Japan, Kanagawa, Japan). Levels of TGF- $\beta 1$ and IGF-1 were measured using commercial ELISA kits as follows; TGF- $\beta 1$ (Quantikine, 2nd Generation (96 well), R\&D Systems, Minneapolis, MN, U.S.A.), IGF-1 (Quantikine (96 well), R\&D Systems, Minneapolis, MN, U.S.A.). Amounts of each protein, type I collagen, TGF- $\beta 1$ and IGF-1, are expressed as values normalized against the protein content of cells determined using a BCA protein assay kit (Thermo Fisher Scientific, Waltham, MA, U.S.A.).

Quantitative PCR Analysis (qPCR) NHDFs were cultured in DMEM containing $0.5 \%$ FBS with or without the PPE at various concentrations for $24 \mathrm{~h}$. Total RNA was extracted using a SuperPrep Cell Lysis \& RT Kit for qPCR (Toyobo, Osaka, Japan) and cDNA was synthesized. qPCR was accomplished using THUNDERBIRD SYBR qPCR mix (Toyobo). A Fast Real-Time PCR System (7900HT, Applied Biosystems, Waltham, MA, U.S.A.) was used to detect qPCR values, and relative quantifications were carried out using the $\Delta \Delta \mathrm{Ct}$ method. The various mRNAs (COL1A1, COL1A2, $\mathrm{T} \beta \mathrm{RI}, \mathrm{T} \beta \mathrm{RII}$ ) and their primers (Prime Time $\mathrm{qPCR}$ primers, Integrated DNA Technologies, Coralville, IA, U.S.A.) are listed in Table 1. Glyceraldehyde-3-phosphate dehydrogenase (GAPDH) was measured as an endogenous control.

Western Blotting NHDFs were cultured in DMEM containing $0.5 \%$ FBS with or without the PPE at various concentrations for $1 \mathrm{~h}$. Afterwards, cells were lysed with lysis buffer $(50 \mathrm{mM}$ Tris- $\mathrm{HCl}, 1 \mathrm{mM}$ EDTA-2Na, $1 \times$ protease inhibitor cocktail, $1 \times$ PhosSTOP, $0.1 \%$ TritonX-100 and $0.1 \%$ sodium dodecyl sulfate (SDS)) to extract proteins. The proteins were separated using 8-16\% Mini-PROTEAN TGX Stain-Free Gels (Bio-Rad, Hercules, CA, U.S.A.) and were then transferred to Immun-Blot LF polyvinylidene difluoride (PVDF) Membranes (Bio-Rad). After blocking with $2 \%$ BSA in Tris-buffered saline containing $0.1 \%$ Tween 20 (TBS-T) at room temperature, the membranes were incubated with the primary antibody at $4^{\circ} \mathrm{C}$ for $24 \mathrm{~h}$ and then were further incubated with the secondary antibody for $1 \mathrm{~h}$. Antibodies bound to proteins on the membranes were visualized with Ez West Blue (Atto, Tokyo,

Table 1. Target Genes and Primer Sequences Used for qPCR

\begin{tabular}{|c|c|}
\hline Gene & Primer sequence \\
\hline COL1A1 & $\begin{array}{l}\text { sense: 5'-GACATGTTCAGCTTTGTGGAC-3' } \\
\text { antisense: 5'-TTCTGTACGCAGGTGATTGG-3' }\end{array}$ \\
\hline COL1A2 & $\begin{array}{l}\text { sense: 5'-GTGAGAGAGGAGTTGTTGGAC-3' } \\
\text { antisense: 5'-CCTTCAATCCATCCAGACCAT-3' }\end{array}$ \\
\hline $\mathrm{T} \beta \mathrm{RI}$ & $\begin{array}{l}\text { sense: 5'-GGTCTTGCCCATCTTCACA-3' } \\
\text { antisense: 5'-GCCAGTCCTAAGTCTGCAAT-3' }\end{array}$ \\
\hline $\mathrm{T} \beta \mathrm{RII}$ & $\begin{array}{l}\text { sense: 5' -TGACAACCAGAAATCCTGCAT-3' } \\
\text { antisense: 5'-CTGTCTCTAGTGTTATGTTCTCGTC-3' }\end{array}$ \\
\hline GAPDH & $\begin{array}{l}\text { sense: 5'-AGGGTGGTGGACCTCAT-3' } \\
\text { antisense: 5'-TGAGTGTGGCAGGGACT-3' }\end{array}$ \\
\hline
\end{tabular}


(A)
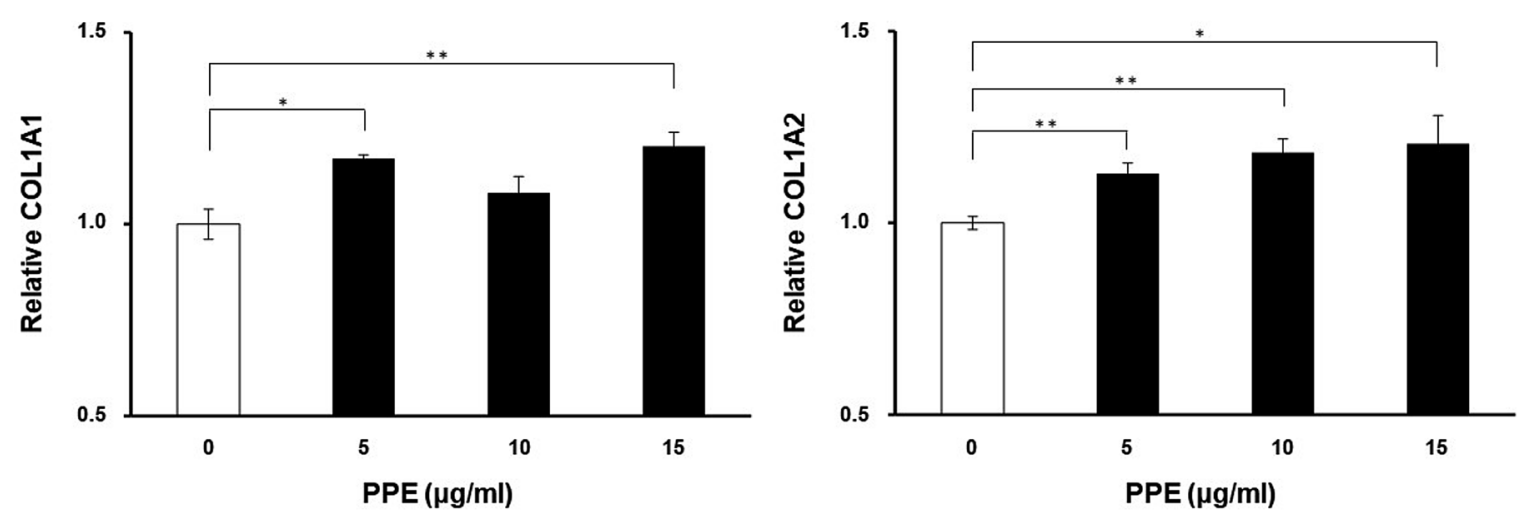

(B)

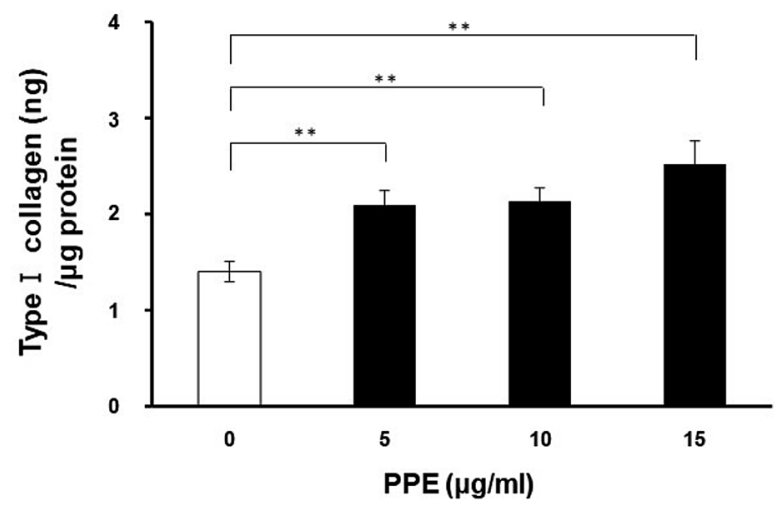

Fig. 1. Effects of the PPE on Type I Collagen Synthesis in NHDFs

NHDFs were incubated with or without various concentrations of the PPE $(0-15 \mu \mathrm{g} / \mathrm{mL})$ for $24 \mathrm{~h}$. (A) mRNA expression levels of COL1A1 and COL1A2 were analyzed by qPCR, and were normalized to the expression of GAPDH. (B) The secretion of type I collagen was analyzed by ELISA. Values represent means \pm S.E.M. of three independent experiments. ${ }^{*} p<0.05,{ }^{*} p<0.01$.

Japan) and levels were measured using a Gel Doc Ez imager (Bio-Rad). $\beta$-Actin was measured as an endogenous control.

Statistical Analysis Measured values are expressed as means \pm standard error of the mean (S.E.M.), and Student's $t$-test was used to identify statistically significant differences. A $p$-value $<0.05$ is considered the threshold for significance.

\section{RESULTS}

Effects of the PPE on the Synthesis of Type I Collagen in NHDFs The effects of the PPE on the synthesis of type I collagen were examined. NHDFs treated with the PPE for $24 \mathrm{~h}$ showed a significant upregulation of mRNA expression levels of COL1A1 and COL1A2 compared to the untreated control (Fig. 1A). Furthermore, treatment of NHDFs with the PPE increased the amount of type I collagen protein in a PPE concentration-dependent manner (Fig. 1B).

Involvement of IGF-1 Signaling and TGF- $\beta$ Signaling on the PPE-Induced Synthesis of Type I Collagen in NHDFs IGF-1 is one of the primary growth factors that induces the synthesis of type I collagen. ${ }^{6}$ Thus, in order to investigate the mechanism involved in the induction of type I collagen synthesis by the PPE, we examined the contribution of IGF-1 signaling. First, we examined whether the PPE stimulates IGF-1 synthesis. After treatment of the PPE $24 \mathrm{~h}$, the amount of IGF-1 in culture supernatants was measured. NHDFs treated with the
PPE $5 \mu \mathrm{g} / \mathrm{mL}$ slightly increased the IGF-1 production, whereas treatment of NHDFs with the PPE $10,15 \mu \mathrm{g} / \mathrm{mL}$ did not have increased in a PPE concentration-dependent manner (Fig. 2A). Then, we examined whether the PPE causes the phosphorylation of IGF-1 receptor (IGF-1R), but increase in the phosphorylation of IGF-1R was not detected (Fig. 2B). From these results, it was thought to be unlikely that the IGF-1 signaling pathway is involved in the PPE-induced synthesis of type I collagen.

Thus, we examined other possible mechanisms that might be involved in the effects of the PPE on NHDFs. It has been reported that TGF- $\beta$ is also a stimulatory factor for type I collagen synthesis. ${ }^{7,8)}$ Thus, we examined the possible contribution of TGF- $\beta$ signaling on the PPE-induced synthesis of type I collagen. First, we examined whether the PPE stimulates TGF- $\beta$ synthesis. NHDFs treated with the PPE for $24 \mathrm{~h}$ did not have increased levels of TGF- $\beta$ (Fig. 3A). We then examined whether the PPE increases levels of the TGF- $\beta$ receptor subunits (T $\beta$ RI and/or T $\beta$ RII) from the aspect of their mRNA expression levels. Treatment with the PPE significantly upregulated the mRNA expression levels of $\mathrm{T} \beta \mathrm{RI}$ and $\mathrm{T} \beta \mathrm{RII}$ (Fig. 3B). This result indicated that the PPE stimulated the synthesis of type I collagen through TGF- $\beta$ signaling due to the upregulation of the TGF- $\beta$ receptor.

PPE-Induced TGF- $\beta$ Signaling in NHDFs Smaddependent and Smad-independent pathways are involved in regulating TGF- $\beta$ signaling. ${ }^{11)}$ Thus, in order to identify the 


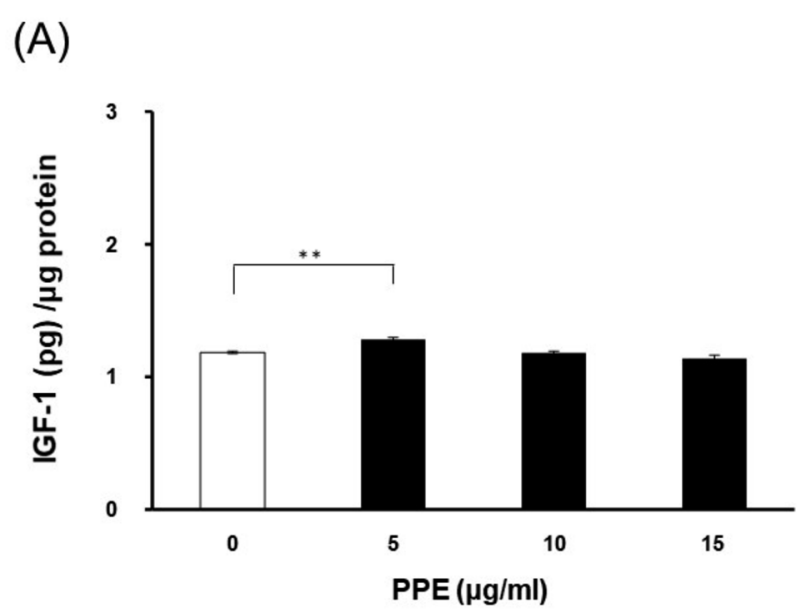

(B)

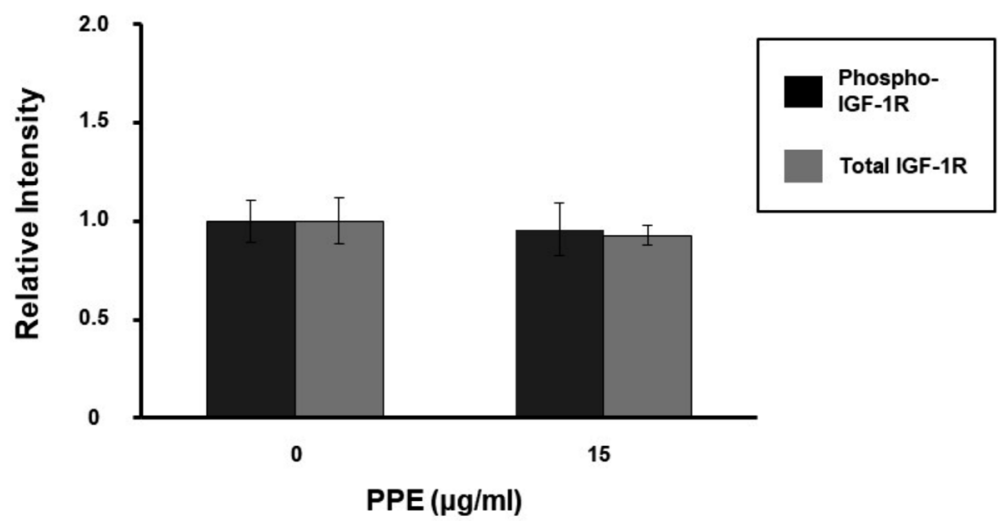

PPE $(\mu \mathrm{g} / \mathrm{ml})$
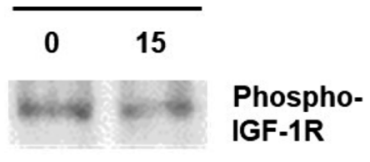

wive row Total IGF-1R

B-Actin

Fig. 2. Effects of the PPE on IGF-1 Signaling in NHDFs

NHDFs were incubated with or without various concentrations of the PPE $(0-15 \mu \mathrm{g} / \mathrm{mL})$ for $24 \mathrm{~h}$. (A) The secretion of IGF-1 was analyzed by ELISA. (B) Cell lysates were analyzed by Western blotting. The densities of IGF-1 receptor (IGF-1R) and phospho-IGF-1R were quantified, and expression levels were normalized to $\beta$-actin. Values represent means \pm S.E.M. of three independent experiments. $* * p<0.01$.

precise mechanism involved, we examined whether the PPE causes the phosphorylation of Smad3, but no increase in the phosphorylation of Smad3 was detected (Fig. 4). That result indicated that the PPE does not function through Smad signaling in collagen synthesis. Previous studies reported that TGF- $\beta$ promotes the production of the extracellular matrix in the dermis through PI3K/Akt and MAPK/ERK signaling. ${ }^{25,26)}$ Thus, we examined the possible involvement of PI3K/Akt and MAPK/ERK signaling in the PPE-induced synthesis of type I collagen. First, we examined whether the PPE causes the phosphorylation of Akt and ERK1/2, the results showed that the PPE significantly increased the phosphorylation of Akt and ERK1/2 (Figs. 5A, B) in NHDFs. Furthermore, we assessed whether LY294002, a specific inhibitor of PI3K that phosphorylates Akt, or U0126, a specific inhibitor of MEK1 and MEK2 that phosphorylates ERK1/2, inhibits PPE-induced type I collagen synthesis. LY294002- or U0126-treated NHDFs significantly inhibited PPE-stimulated increase of type I collagen (Fig. 5C). From these results, we concluded that the PPE promotes type I collagen synthesis by activating PI3K/Akt and MAPK/ERK signaling.

\section{DISCUSSION}

Collagen produced in dermal fibroblasts forms fibers in the dermis, which provides the mechanical strength of the skin. In aged skin, which is characterized by wrinkles and sagging and the loss of viscoelasticity, a drastic loss of collagen fibers in the papillary dermis is observed as a typical histological alteration. Thus, it is currently accepted that the loss of collagen fibers in the dermis is one cause of wrinkling and sagging of the skin. The loss of collagen fibers in aged skin is considered to be due, at least in part, to an imbalance of collagen synthesis and degradation by MMP-1. Thus, the promotion of collagen synthesis in dermal fibroblasts is considered as an effective approach to prevent and/or improve the symptoms of skin aging. In this study, we examined the effects of a PPE on collagen synthesis as a candidate anti-aging material, because potato peels contain phenolic compounds and flavonoids and also several other compounds that have been reported to enhance collagen synthesis in fibroblasts. ${ }^{17,19)}$

In fact, the results show that the PPE stimulates type I collagen synthesis in NHDFs. Type I collagen synthesis has been demonstrated to be regulated by various growth factors, cytokines and chemokines. ${ }^{27)}$ IGF-1 and TGF- $\beta$ have been reported as predominant factors that promote collagen synthesis. ${ }^{6-8)}$ Treatment of NHDFs with the PPE did not affect the expression of TGF- $\beta$ but showed a significant upregulation of T $\beta$ RI and T $\beta$ RII mRNA levels (Fig. 3). The involvement of the TGF- $\beta$ pathways on collagen synthesis have been 


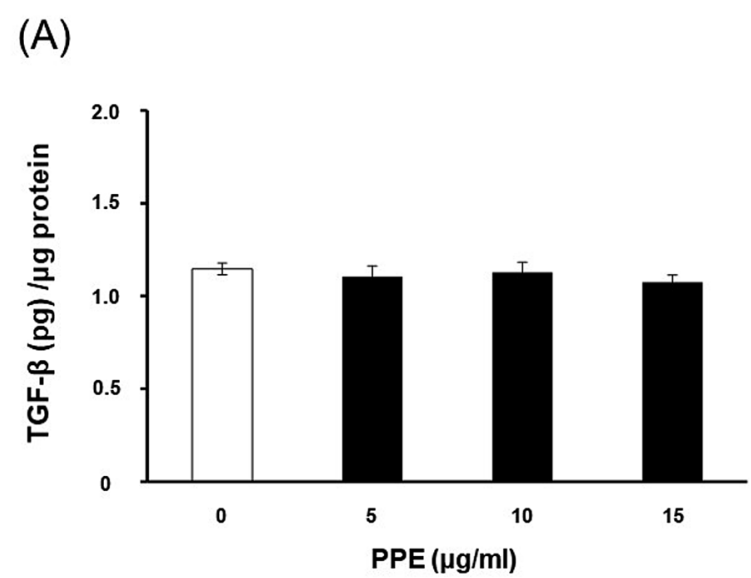

(B)
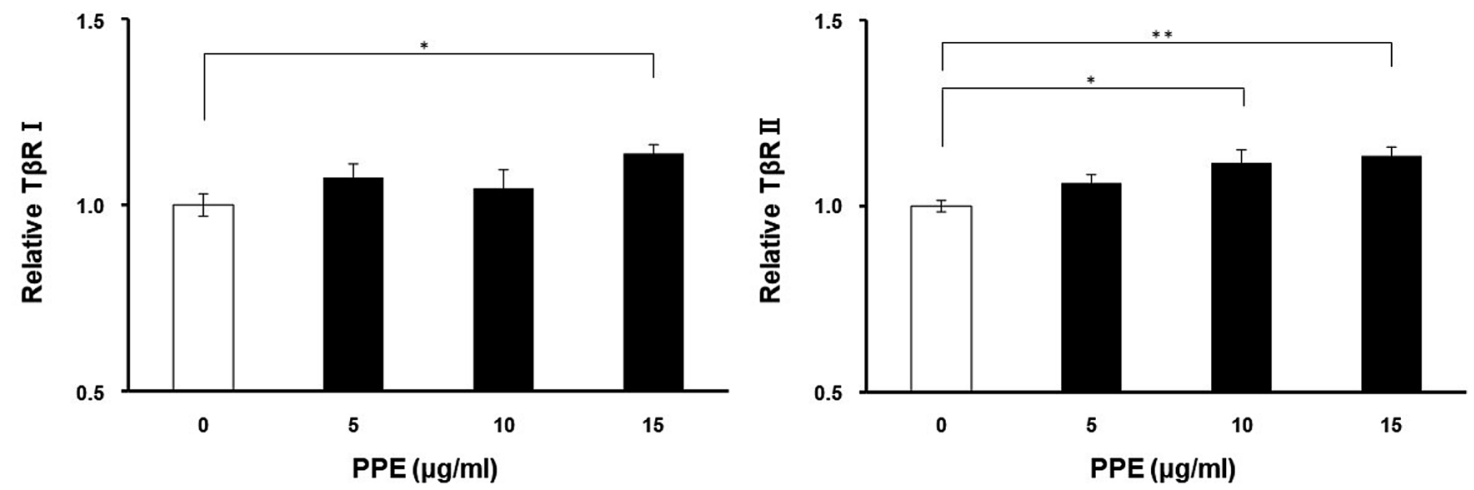

Fig. 3. Effects of the PPE on TGF- $\beta$ Signaling in NHDFs

NHDFs were incubated with or without various concentrations of the PPE $(0-15 \mu \mathrm{g} / \mathrm{mL})$ for $24 \mathrm{~h}$. (A) The secretion of TGF- $\beta$ was analyzed by ELISA. (B) mRNA expression levels of T $\beta$ RI and T $\beta$ RII were analyzed by qPCR, and were normalized to the expression of GAPDH. Values represent means \pm S.E.M. of three independent experiments. $* p<0.05, * * p<0.01$.
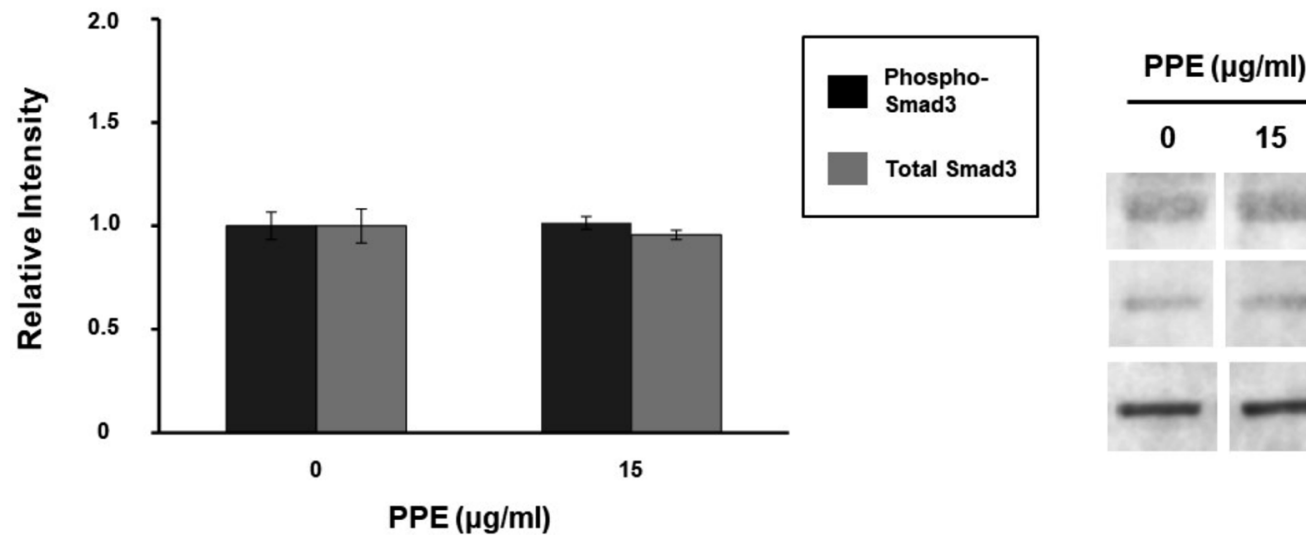

$0 \quad 15$

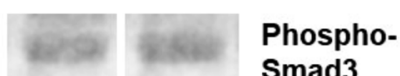

Smad3

Total Smad3

$\beta-A c t i n$

Fig. 4. Effects of the PPE on the Phosphorylation of Smad3

Serum-starved NHDFs were treated with or without the PPE for 1 h. Cell lysates were analyzed by Western blotting. The densities of Smad 3 and phospho-Smad 3 were quantified, and expression levels were normalized to $\beta$-actin. Values represent means \pm S.E.M. of three independent experiments.

reported to involve the Smad-dependent pathway and the Smad-independent pathway by the activation of PI3K/Akt and MAPK/ERK.$^{11,13)}$ To demonstrate the precise mechanism, the involvement of TGF- $\beta$ signaling on the PPE-induced synthesis of type I collagen was examined. The Smad pathway is wellknown to involve TGF- $\beta$ signaling in the stimulation of collagen synthesis. ${ }^{2,7)}$ However, because the PPE failed to stimulate the phosphorylation of Smad (Fig. 4), the contribution of the Smad pathway was ruled out regarding the stimulation of collagen synthesis.
Thus, we shifted to investigating another mechanism, the Smad-independent pathway. It has been reported that TGF- $\beta$ also promotes collagen synthesis by activating PI3K/Akt signaling. ${ }^{13)}$ Knock-down of Akt in dermal fibroblasts decreased collagen synthesis and increased MMP-1. ${ }^{25)}$ Although MAPK/ERK signaling is also controlled by TGF- $\beta$, the response to TGF- $\beta$ is interestingly different depending on the cell type, epithelial cells and mesenchymal cells. ${ }^{8,11,13)}$ TGF- $\beta$ activates ERK signaling in dermal fibroblasts, which are mesenchymal cells, but suppresses it in keratinocytes, which are 
(A)
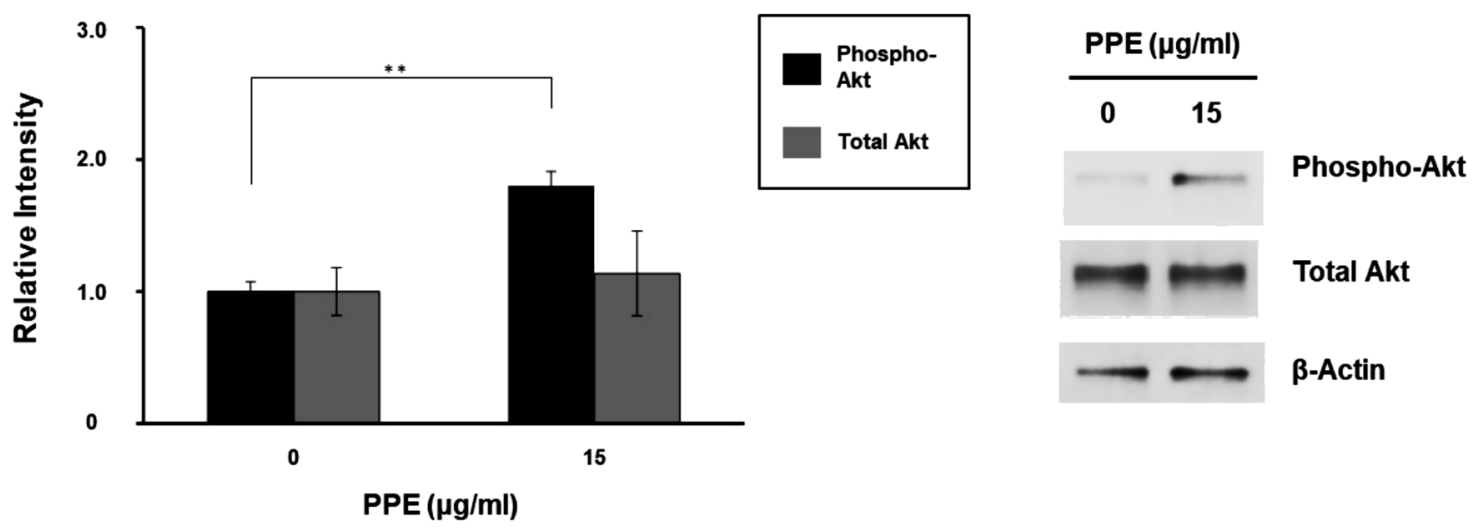

(B)
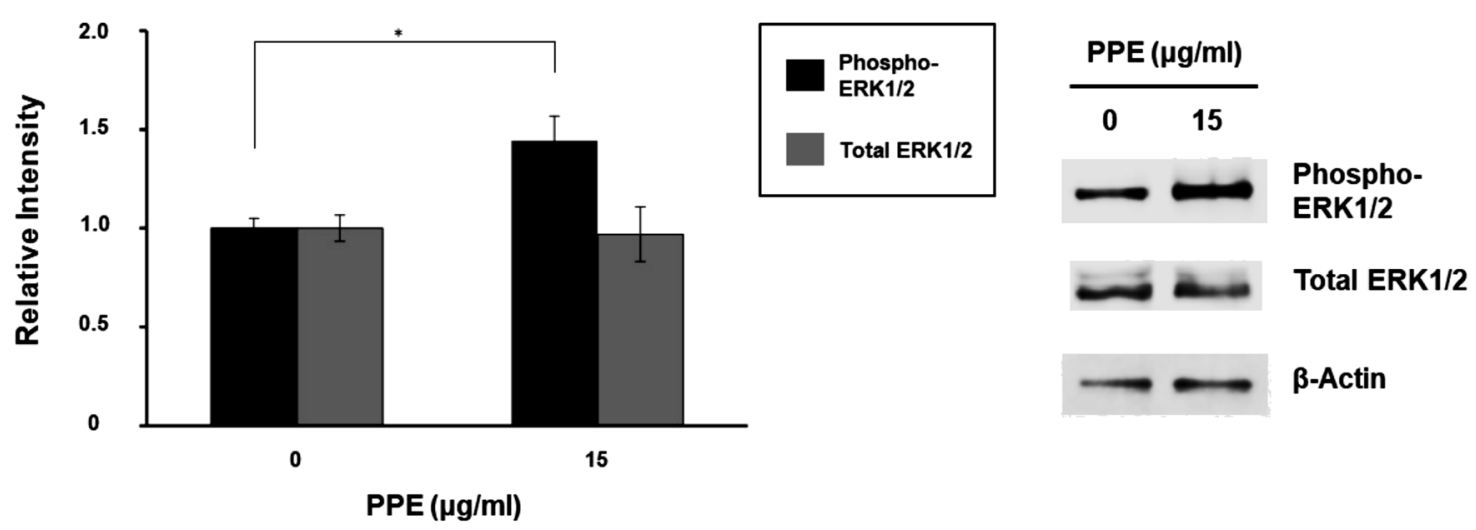

(C)
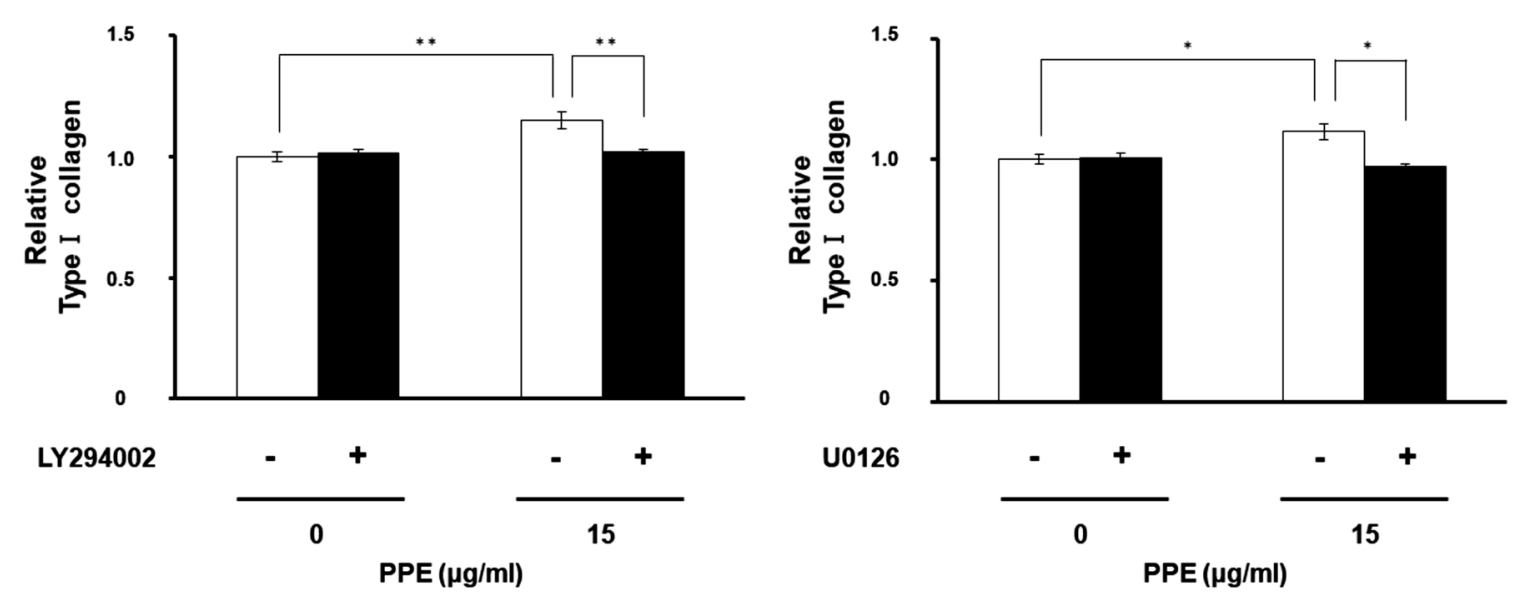

Fig. 5. Effects of the PPE on PI3K/Akt and MAPK/ERK Signaling

(A) (B) Serum-starved NHDFs were treated with or without the PPE for $1 \mathrm{~h}$. Cell lysates were analyzed by Western blotting. The densities of Akt, phospho-Akt, ERK1/2 and phospho-ERK1/2 were quantified, and expression levels were normalized to $\beta$-Actin. (C) Effect of Akt and ERK1/2 signaling inhibitor on PPE-induced collagen synthesis. Serum-starved NHDFs were treated with inhibitor $(10 \mu \mathrm{M})$ or vehicle for $1 \mathrm{~h}$ or $2 \mathrm{~h}$, and then NHDFs were incubated with PPE for $24 \mathrm{~h}$. The secretion of type I collagen was analyzed by ELISA. Values represent means \pm S.E.M. of three independent experiments. ${ }^{*} p<0.05, * * p<0.01$.

epithelial cells. ${ }^{12)}$ This difference in responses is understood by differences in the expression levels of TGF- $\beta$ receptors. TGF- $\beta$ receptors are composed of $\mathrm{T} \beta \mathrm{RI}$ and $\mathrm{T} \beta \mathrm{RII}$. Whereas the expression of $\mathrm{T} \beta \mathrm{RI}$ does not depend on the cell type, $\mathrm{T} \beta \mathrm{RII}$ is expressed at a higher level in dermal fibroblasts compared with keratinocytes. ${ }^{26)}$ On the other hand, the TGF- $\beta$-mediated activation of ERK signaling promoted collagen synthesis in human mesangial cells and human osteoblasts. ${ }^{28,29)}$ It has also been reported that a velvet antler extract, which is known to activate ERK, promotes type I collagen synthesis in human dermal fibroblasts. ${ }^{30)}$ In our study, treatment with the PPE increased expression levels of T $\beta$ RII mRNA (Fig. 3B). PPEtreated NHDFs showed increased phosphorylation of Akt and ERK1/2 (Fig. 5), which indicated that the PPE induced stimulation of collagen synthesis occurred through the activation of PI3K/Akt and MAPK/ERK. PI3K/Akt and MAPK/ERK 
signaling is in the Smad-independent pathway, but there have been few reports on the stimulation of collagen synthesis via these pathways. ${ }^{30,31)}$ Given this, we believe that the PPE is an incredibly useful substance.

It is known that PI3K/Akt and MAPK/ERK signaling is not only involved in regulating collagen synthesis but also affects all aspects of skin aging. ${ }^{32)}$ Decreases of MAPK/ERK signaling are observed in the skin of elderly humans and such declines are particularly evident in photoaged skin. ${ }^{33,34)}$ Furthermore, it has been reported that blocking PI3K/Akt signaling occurs in the senescent phenotype in skin-derived precursor cells. These facts suggest that PI3K/Akt signaling plays a critical role to prevent the aging process. ${ }^{35)}$

Gathering these facts, we conclude that the PPE, which stimulates collagen synthesis in NHDFs through the activation of PI3K/Akt and MAPK/ERK signaling, will be a novel and effective antiaging material.

Conflict of Interest Mari Suto, Hirofumi Masutomi and Katsuyuki Ishihara are employees of Calbee, Inc. Hitoshi Masaki has no conflict of interest.

\section{REFERENCES}

1) Brett D. A review of collagen and collagen-based wound dressings. Wounds, 20, 347-356 (2008)

2) Teti A. Regulation of cellular functions by extracellular matrix. $J$. Am. Soc. Nephrol., 2 (Supp1.), S83-S87 (1992).

3) Sachs DL, Voorhees JJ. Age-reversing drugs and devices in dermatology. Clin. Pharmacol. Ther., 89, 34-43 (2011).

4) Chung JH, Seo JY, Choi HR, Lee MK, Youn CS, Rhie G, Cho KH, Kim KH, Park KC, Eun HC. Modulation of skin collagen metabolism in aged and photoaged human skin in vivo. J. Invest. Dermatol., 117, 1218-1224 (2001).

5) Gelse K, Pöschl E, Aigner T. Collagens-structure, function, and biosynthesis. Adv. Drug Deliv. Rev., 55, 1531-1546 (2003).

6) Gillery P, Leperre A, Maquart FX, Borel JP. Insulin-like growth factor-1 (IGF-1) stimulates protein synthesis and collagen gene expression in monolayer and lattice cultures of fibroblasts. $J$. Cell. Physiol., 152, 389-396 (1992).

7) Verrecchia F, Mauviel A. Transforming growth factor- $\beta$ signaling through the smad pathway: role in extracellular matrix gene expression and regulation. J. Invest. Dermatol., 118, 211-215 (2002).

8) Leask A, Abraham DJ. TGF- $\beta$ signaling and the fibrotic response. FASEB J., 18, 816-827 (2004).

9) Ryan PD, Goss PE. The emerging role of the insulin-like growth factor pathway as a therapeutic target in cancer. Oncologist, 13, 16-24 (2008)

10) Yin M, Guan $X$, Liao Z, Wei Q. Insulin-like growth factor-1 receptor-targeted therapy for non-small cell lung cancer: a mini review. Am. J. Transl. Res., 1, 101-114 (2009).

11) Derynck R, Zhang YE. Smad-dependent and smad-independent pathways in TGF- $\beta$ family signaling. Nature, 425, 577-584 (2003).

12) Nakerakanti S, Trojanowska M. The role of TGF- $\beta$ receptors in fibrosis. Open Rheumat. J., 6, 156-162 (2012).

13) Zhang YE. Non-smad signaling pathways of the TGF- $\beta$ family. Cold Spring Harb. Perspect. Biol., 9, a022129 (2017).

14) Dale MFB, Griffiths DW, Todd DT. Effect of genotype, environment, and postharvest storage on the total ascorbate content of potato ( $\mathrm{So}-$ lanum tuberosum) tubers. J. Agric. Food Chem., 51, 244-248 (2003).

15) Goyer A, Navarre DA. Determination of folate concentrations in diverse potato germplasm using a trienzyme extraction and a microbiological assay. J. Agric. Food Chem., 55, 3523-3528 (2007).

16) Camire ME, Kubow S, Donnelly DJ. Potatoes and human health.
Crit. Rev. Food Sci. Nutr., 49, 823-840 (2009).

17) Ezekiel R, Singh N, Sharma S, Kaur A. Beneficial phytochemicals in potato-a review. Food Res. Int., 50, 487-496 (2013).

18) Brown CR. Antioxidants in potato. Am. J. Potato Res., 82, 163-172 (2005).

19) Nara K, Miyoshi T, Honma T, Koga H. Antioxidative activity of bound-form phenolics in potato peel. Biosci. Biotechnol. Biochem., 70, 1489-1491 (2006).

20) Murad S, Grove D, Lindberg KA, Reynolds G, Sivarajah A, Pinnell SR. Regulation of collagen synthesis by ascorbic acid. Proc. Natl. Acad. Sci. U.S.A., 78, 2879-2882 (1981).

21) Działo M, Mierziak J, Korzun U, Preisner M, Szopa J, Kulma A. The potential of plant phenolics in prevention and therapy of skin disorders. Int. J. Mol. Sci., 17, 160 (2016).

22) Hahn HJ, Kim KB, Bae S, Choi BG, An S, Ahn KJ, Kim SY. Pretreatment of ferulic acid protects human dermal fibroblasts against ultraviolet A irradiation. Ann. Dermatol., 28, 740-748 (2016).

23) Wu PY, Huang CC, Chu Y, Huang YH, Lin P, Liu YH, Wen KC, Lin CY, Hsu MC, Chiang HM. Alleviation of ultraviolet B-induced photodamage by Coffea arabica extract in human skin fibroblasts and hairless mouse skin. Int. J. Mol. Sci., 18, 782 (2017).

24) Kim J, Hwang JS, Cho YK, Han Y, Jeon YJ, Yang KH. Protective effects of (-)-epigallocatechin-3-gallate on UVA and UVB-induced skin damage. Skin Pharmacol. Appl. Skin Physiol., 14, 11-19 (2001).

25) Bujor AM, Pannu J, Bu S, Smith EA, Muise-Helmericks RC, Trojanowska M. Akt blockade downregulates collagen and upregulates MMP1 in human dermal fibroblasts. J. Invest. Dermatol., 128, 1906-1914 (2008).

26) Bandyopadhyay B, Han A, Dai J, Fan J, Li Y, Chen M, Woodley DT, Li W. T $\beta$ RI/Alk5-independent T $\beta$ RII signaling to ERK1/2 in human skin cells according to distinct levels of T $\beta$ RII expression. $J$. Cell Sci., 124, 19-24 (2011).

27) Kim MS, Song HJ, Lee SH, Lee CK. Comparative study of various growth factors and cytokines on type I collagen and hyaluronan production in human dermal fibroblasts. J. Cosmet. Dermatol., 13, 44-51 (2014).

28) Hayashida T, Poncelet AC, Hubchak SC, Schnaper HW. TGF- $\beta 1$ activates MAP kinase in human mesangial cells: A possible role in collagen expression. Kidney Int., 56, 1710-1720 (1999).

29) Palcy S, Goltzman D. Protein kinase signaling pathways involved in the up-regulation of the rat $\alpha 1$ (I) collagen gene by transforming growth factor $\beta 1$ and bone morphogenetic protein 2 in osteoblastic cells. Biochem. J., 343, 21-27 (1999).

30) Roh SS, Lee MH, Hwang YL, Song HH, Jin MH, Park SG, Lee CK, Kim CD, Yoon TJ, Lee JH. Stimulation of the extracellular matrix production in dermal fibroblasts by velvet antler extract. Ann. Dermatol., 22, 173-179 (2010).

31) Takasao N, Tsuji-Nait K, Ishikura S, Tamura A, Akagawa M. Cinnamon extract promotes type I collagen biosynthesis via activation of IGF-1 signaling in human dermal fibroblasts. J. Agric. Food Chem., 60, 1193-1200 (2012).

32) Lorenzini A, Tresini M, Mawal-Dewan M, Frisoni L, Zhang H, Allen RG, Sell C, Cristofalo VJ. Role of the Raf/MEK/ERK and the PI3K/Akt (PKB) pathways in fibroblast senescence. Exp. Gerontol., 37, 1149-1156 (2002).

33) Chung JH, Kang S, Varani J, Lin J, Fisher GJ, Voorhees JJ. Decreased extracellular-signal-regulated kinase and increased stressactivated MAP kinase activities in aged human skin in vivo. $J$. Invest. Dermatol., 115, 177-182 (2000).

34) Shin MH, Rhie G, Kim YK, Park CH, Cho KH, Kim KH, Eun HC, Chung JH. $\mathrm{H}_{2} \mathrm{O}_{2}$ accumulation by catalase reduction changes MAP kinase signaling in aged human skin in vivo. J. Invest. Dermatol., 125, 221-229 (2005).

35) Liu S, Liu S, Wang X, Zhou J, Cao Y, Wang F, Duan E. The PI3K/ Akt pathway inhibits senescence and promotes self-renewal of human skin-derived precursors in vitro. Aging Cell, 10, 661-674 (2011). 